\section{Two-dimensional crystallization}

SIR - Many people have obtained two-dimensional (2D) colloid crystals on substrates ${ }^{1-4}$, but they have observed the final results of ordering and have not investigated the mechanism of this process. We have directly observed the dynamics of $2 \mathrm{D}$ array formation of latex particles on solid substrate by means of optical microscopy. Our observations suggest a two-stage mechanism of $2 \mathrm{D}$ crystallization: (1) Nucleus formation, governed by attractive capillary forces appearing between particles partially immersed in a liquid layer ${ }^{5}$; and (2) Crystal growth, through con-

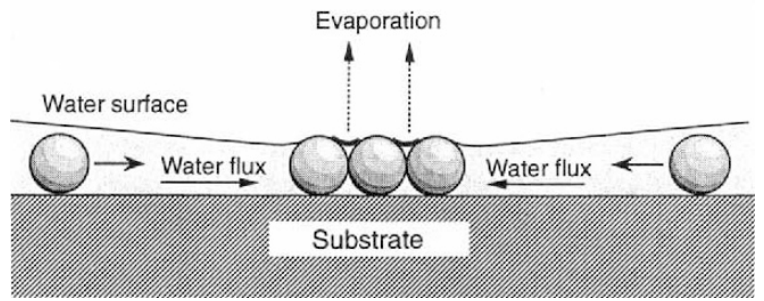

FIG. 2 Schematic presentation of the particle assembly process driven by the liquid flow.

vective particle flux caused by the water evaporation from the already ordered array.

A fast and convenient method for formation of a $2 \mathrm{D}$ protein array on a mercury surface has been recently developed ${ }^{2.3}$. The good quality of the samples thus obtained allowed investigation of the protein orientation and structure by electron microscopy combined with image reconstruction. Highly ordered $2 \mathrm{D}$ crystals can thus be obtained in a controllable way; this technique could lead to further development of the
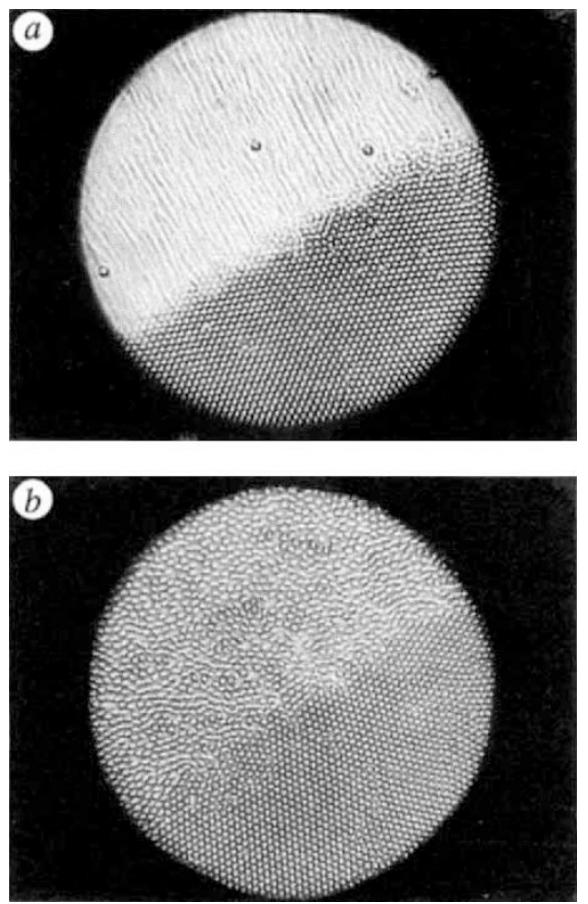

FIG. 1 Photographs of 2D-crystal growth: a, tracks of the particles rushing towards the ordered phase; $b$, at decreased rate of water evaporation the velocity of the moving particles is lower and the tracks are shorter. controlled building up of well-ordered protein monolayers and multilayers - a possible step towards a future high technology at the macromolecular level ${ }^{3}$. 2D arrays on solid substrates can find applications in some modern techniques, such as data storage, optical devices and microelectronics ${ }^{4}$

In our model experiments, we investigated the mechanism of $2 \mathrm{D}$ crystallization using a suspension containing monodisperse particles of $1.70 \mu \mathrm{m}$ diameter.

\section{vaporation}

This was spread over a horizontal hydrophilic glass plate encircled by a teflon ring. The slightly concave layer formed gradually thins owing to the water evaporation; when its thickness in the centre of the substrate becomes equal to the particle diameter, a nucleus of $2 \mathrm{D}$ crystal suddenly forms. The particles in the thicker layer encircling the nucleus begin to move towards the ordered zone and upon reaching the boundary of the array they are trapped in it (Fig. 1a). In some experiments we added $0.2 \mathrm{wt} \%$ glucose to the suspension and the flux of particles became slower (Fig. 1b).

The nature of the forces governing the ordering is revealed by the fact that in all experiments the $2 \mathrm{D}$ crystallization always started when the thickness of the water layer became equal to the particle diameter. This implies that the 2Dcrystal nuclei are formed under the capillary attraction arising when the tops of the particles protrude from the water layer (Fig. 2). The attraction energy can be much larger than the thermal energy (kT) even with nanometre-sized particles ${ }^{5}$.

We were able to show that the crystal growth is caused by a convective transport of particles towards the ordered nucleus. This effect appears when menisci (shown bold in Fig. 2) form around the protruding tops of the hydrophilic particles in the nucleus. These menisci hinder the further thinning of the water layer in the nucleus. An intensive water influx from the thicker parts of the layer,

1. Alfrey, T. Jr, Bradford, E. B. \& Vanderhoff, J. W. J. Opt Soc. Am. 44, 603-609 (1954)

Yoshimura, H., Matsumoto, M., Endo, S. \& Nagayama, K. Uitramicroscopy 32, 265-274 (1990).

3. Nagayama, K. Nanobiology 1, 25-37 (1992). J. Colloid Interface Sci. 144, 538-547 (1991).

5. Kralchevsky, P. A., Paunov, V. N., Ivanov, I. B. \& Nagayama, K. J. Colloid Interface Sci. 151, 79-94 (1992).
4. Hayashi, S., Kumamoto, Y., Suzuki, T. \& Hirai, T. which tends to compensate the water evaporation from the nucleus, appears next. This flux carries the suspended particles towards the nucleus. By decreasing or increasing the water evaporation rate we could speed up or slow down the convective particle transport. At increased humidity, we saw a complete arrest of the process of ordering and even disintegration of the already ordered clusters.

N. D. Denkov

O. D. Velev

P. A. Kralchevsky

I. B. Ivanov*

Faculty of Chemistry,

University of Sofia,

1126 Sofia,

Bulgaria

H. Yoshimura

K. Nagayama

Protein Array Project, ERATO,

JRDC, 18-1 Higashiarai,

Tsukuba,

305 Japan

\footnotetext{
* To whom correspondence should be addressed.
}

\section{Checkpoint check}

SIR - Andrew Murray in his interesting and comprehensive Review Article (Nature 359, 599-604; 1992) misnames the fission-yeast checkpoint genes that we have identified (Fig. 3). Our paper that is cited in support of the information in fact reported mutants rather than genes. These mutants define five new checkpoint genes called hus1-hus5. Mutations of any one of the genes prevent arrest in response to inhibition of DNA replication and in addition cause increased sensitivity to radiation. Thus it is unlikely that any one of them is involved solely in detection of unreplicated DNA as Murray's Fig. 3 suggests. A full report on our work has appeared in Genes and Development $(\mathbf{6}, 2035$ 2046; 1992).

Tamar Enoch

Anthony Carr

Paul Nurse

ICRF Cell Cycle Group,

Department of Biochemistry,

University of Oxford,

Oxford OX1 3QU, UK

\section{Scientific Correspondence}

Scientific Correspondence is a relatively informal section of Nature in which matters of general scientific interest, not necessarily those arising from papers appearing in Nature, are published. Because there is space to print only a small proportion of the letters received, priority is usually given according to general interest and topicality, to contributions of fewer than 500 words, and to contributions using simple language. 